Published in final edited form as:

Int J Cardiol. 2013 September 30; 168(2): . doi:10.1016/j.ijcard.2012.12.103.

\title{
PREDICTIVE VALUE OF NIGHT-TIME HEART RATE FOR CARDIOVASCULAR EVENTS IN HYPERTENSION. THE ABP- INTERNATIONAL STUDY
}

\author{
Paolo Palatini, MD1 ${ }^{1}$, Gianpaolo Reboldi, MD, PhD, MSc ${ }^{2}$, Lawrence J Beilin, MD³, Kazuo \\ Eguchi, MD ${ }^{4}$, Yutaka Imai, MD ${ }^{5}$, Kazuomi Kario, MD ${ }^{4}$, Takayoshi Ohkubo, MD ${ }^{5,6}$, Sante D \\ Pierdomenico, MD7, Francesca Saladini, MD ${ }^{1}$, Joseph E Schwartz, PhD ${ }^{8,9}$, Lindon Wing, \\ MB, FRACP ${ }^{10}$, and Paolo Verdecchia, MD ${ }^{11}$ \\ 1 University of Padova, Padua, Italy \\ ${ }^{2}$ University of Perugia, Perugia, Italy \\ 3 University of Western Australia, Perth, Australia \\ ${ }^{4}$ Jichi University, Tochigi, Japan \\ ${ }^{5}$ Tohoku University, Sendai, Japan \\ ${ }^{6}$ Shiga University of Medical Science, Otsu, Japan \\ ${ }^{7}$ University of Chieti, Chieti, Italy \\ ${ }^{8}$ Columbia University, New York, USA \\ ${ }^{9}$ Stony Brook University, New York, USA \\ ${ }^{10}$ Flinders University, Adelaide, Australia \\ ${ }^{11}$ Hospital of Assisi, Italy
}

\section{Abstract}

Background-Data from prospective cohort studies regarding the association between ambulatory heart rate (HR) and cardiovascular events (CVE) are conflicting.

Methods-To investigate whether ambulatory HR predicts CVE in hypertension, we performed 24-hour ambulatory blood pressure and HR monitoring in 7600 hypertensive patients aged 52 \pm 16 years from Italy, U.S.A., Japan, and Australia, included in the 'ABP-International' registry. All were untreated at baseline examination. Standardized hazard ratios for ambulatory HRs were computed, stratifying for cohort, and adjusting for age, gender, blood pressure, smoking, diabetes, serum total cholesterol and serum creatinine.

\footnotetext{
(C) 2012 Elsevier Ireland Ltd. All rights reserved.

Correspondence to: Prof. Paolo Palatini, M.D., Department of Medicine, Via Giustiniani, 235128 Padova (Italy), Phone: +39-049-8212278, Fax: +39-049-8754179, palatini@unipd.it.

$1-11$ This author takes responsibility for all aspects of the reliability and freedom from bias of the data presented and their discussed interpretation

Conflicts of interest: none

Publisher's Disclaimer: This is a PDF file of an unedited manuscript that has been accepted for publication. As a service to our customers we are providing this early version of the manuscript. The manuscript will undergo copyediting, typesetting, and review of the resulting proof before it is published in its final citable form. Please note that during the production process errors may be discovered which could affect the content, and all legal disclaimers that apply to the journal pertain.
} 
Results-During a median follow-up of 5.0 years there were 639 fatal and nonfatal CVE. In a multivariable Cox model, night-time HR predicted fatal combined with nonfatal CVE more closely than $24 \mathrm{~h}$ HR ( $\mathrm{p}=0.007$ and $=0.03$, respectively). Daytime HR and the night:day HR ratio were not associated with $\operatorname{CVE}$ ( $\mathrm{p}=0.07$ and $=0.18$, respectively). The hazard ratio of the fatal combined with nonfatal CVE for a 10-beats/min increment of the night-time HR was 1.13 (95\% CI, 1.04-1.22). This relationship remained significant when subjects taking beta-blockers during the follow-up (hazard ratio, 1.15; 95\% CI, 1.05-1.25) or subjects who had an event within 5 years after enrollment (hazard ratio, 1.23; 95\% CI, 1.05-1.45) were excluded from analysis.

Conclusions-At variance with previous data obtained from general populations, ambulatory HR added to the risk stratification for fatal combined with nonfatal CVE in the hypertensive patients from the ABP-International study. Night-time HR was a better predictor of CVE than daytime HR.

\section{Keywords}

ambulatory; sleep; heart rate; cardiovascular; events; hypertension

\section{INTRODUCTION}

Several studies have demonstrated an association between resting heart rate (HR) and cardiovascular mortality both in general populations (1-3) and in patients with hypertension $(4,5)$ or cardiac diseases $(6,7)$. However, a few HR readings taken in a clinic environment might be poorly representative of the actual HR levels leading to an underestimation of their real predictive power. HR assessed out of the office in association with ambulatory blood pressure is more representative of a subject's usual HR and offers additional information about the HR circadian pattern, including HR during the sleep period. Several studies have specifically explored the association between ambulatory HR and fatal and nonfatal cardiovascular events (CVE) but the evidence relating outcome to ambulatory HR remains incomplete and lacks consistency (8-14). Most investigators reported only on fatal outcomes, with night-time HR or a blunted fall in HR at night predicting all-cause mortality in some of these studies $(8,9,12,13)$. HR nondipping predicted CVE in one study (14). In keeping with what was observed with ambulatory blood pressure (15), these results suggest that HR during sleep, or the day-night HR dip might have better predictive power than daytime HR. The few studies that reported information on CVE failed to demonstrate an independent association between ambulatory HR and cardiovascular outcomes either in general populations $(12,13)$ or in hypertensive patients $(8)$. Establishing the predictive value of ambulatory HR for CVE in hypertension would have important therapeutic implications because antihypertensive drugs with cardiac slowing properties may exert an additional benefit in patients with high HR.

The "Ambulatory Blood Pressure in referred hypertensive subjects: an International database" (ABP-International) is an international consortium composed of normotensive and hypertensive subjects assessed with 24-hour ambulatory blood pressure and HR measurement. The aim of the current study was to explore the relationship between ambulatory HR at baseline and fatal combined with nonfatal CVE in this international database and to investigate the relationship between 24-hour, daytime, and night-time HR and CVE in the 7600 hypertensive patients from this database. 


\section{METHODS}

\section{Study population}

The ABP-International study is an international registry established in order to investigate the relationship of 24-hour ambulatory blood pressure and HR with morbidity and mortality in a large patient population recruited in different centers around the world. The database was constructed by systematically merging 8 prospective studies from 3 European, 3 Japanese, 1 North American, and 1 Australian centers. Although protocols were not standardized, studies were conducted following similar procedures and were eligible for inclusion if they involved a random population sample or patients referred to hospital for hypertension, if baseline information on ambulatory blood pressure and HR and cardiovascular risk factors was available, and if the follow-up included fatal and nonfatal CVE. The above information was provided by the investigators in electronic format. For the present analysis, we selected untreated participants in which office and ambulatory HRs were available and office blood pressure was in the hypertensive range $(\geq 140 \mathrm{mmHg}$ systolic or $\geq 90 \mathrm{mmHg}$ diastolic). Thus, 3635 subjects were excluded because of missing data on relevant variables, antihypertensive treatment at baseline, or office blood pressure < 140/90 $\mathrm{mmHg}$ (figure 1) leaving 6 cohorts (office HR and/or ambulatory HR not available in the Ohasama study and the American Study) and 7600 participants for analysis. Thus, the present analysis included 2942 participants from the PIUMA study, Italy (16), 671 patients from the Jichi School of Medicine, Japan (17), 1859 patients from the University of Chieti, Italy (18), 664 participants from the ANBP2 study, Australia (19), 346 patients from one clinic and two hospitals in the Karatsu-Nishiarita Study, Japan (20), and 1118 participants from the HARVEST study, Italy (21). Study protocols were approved by the local Ethics Committees and informed consent was obtained from all of the participants. The study procedures were conducted in accordance with the ethical guidelines of the 1975 Declaration of Helsinki and the local institutional guidelines.

\section{Blood pressure and heart rate measurements}

Office blood pressure was measured by trained observers using a standard auscultatory method and was the mean of 2 to 6 readings. Office HR was measured from pulse palpation over $\geq 15$ seconds, ranging from 15 to 60 seconds, and was the mean of 1 to 3 measurements. For ambulatory monitoring, validated monitors were programmed to obtain ambulatory blood pressure and HR readings at intervals ranging from 10 to 30 minutes during daytime and from 15 to 30 minutes at night. Participant's awake (or daytime) and asleep (or nighttime) periods were determined from the diary sheet in 5 cohorts (mean \pm SD for night-time, 7.1 1.7 hours) and was the interval between 7:00 AM and 11:00 PM and between 11:00 PM and 7:00 AM, respectively, in one study (21). Within individual subjects, the arithmetic average of the 24-hour, daytime and night-time periods were used as the ambulatory measurements. Daytime blood pressure and HR were calculated from an average of $71.3 \pm 22.5$ readings and night-time blood pressure and HR from an average of 16.5 \pm 4.0 readings. The nocturnal fall in blood pressure and HR was computed from the night:day ratio.

\section{Procedures}

Each patient's medical history including intake of medications, and information on smoking and alcohol consumption was collected through questionnaires originally administered in each cohort (22). Current smokers were those who reported smoking one or more cigarettes per day. Alcohol intake was calculated by summing the total number of milliliters of alcohol consumed as wine, beer, and spirits. Subjects were then categorized as nondrinkers (class 0), light drinkers (class 1), moderate drinkers (class 2), and heavy drinkers (class 3 ). Body mass index was body weight in kilograms divided by height in meters squared. Serum creatinine, 
total cholesterol and blood glucose concentrations were measured by automated enzymatic methods. Diabetes mellitus was defined as a self reported diagnosis, the use of antidiabetic drugs, a fasting blood glucose concentration of at least $7.0 \mathrm{mmol} / 1$, or a random blood glucose concentration of at least $11.1 \mathrm{mmol} / \mathrm{l}$. Having diabetes was scored 1 and the absence of diabetes was scored 0 .

The observation period was considered as the number of years from the first available 24hour recording to the date of the last clinical visit or the first endpoint. Information on type of antihypertensive treatment taken during the follow-up was collected. The primary endpoint was a composite of all fatal and first nonfatal CVE. In all outcome analyses, we considered only the first event within each category.

\section{Definition of cardiovascular events}

Cardiovascular events included fatal and nonfatal acute myocardial infarction, sudden cardiac death, new-onset heart failure needing at least hospitalization, death from progressive heart failure or other cardiovascular causes, any myocardial revascularization procedure, fatal and nonfatal strokes, any aortic or lower limb revascularization procedure. Nonfatal stroke did not include transient ischemic attack. We ascertained vital status and the incidence of fatal and nonfatal events from medical records, death certificates and interviews with attending physicians and patient's families.

\section{Statistical Methods}

Statistical analysis was carried out using SYSTAT version 10 and version 11 (SPSS Inc., Chicago, IL, USA), and SAS 9.3 (SAS Institute Inc., Cary, NC, USA) packages. Differences in mean values were tested with an unpaired Student $t$ test. Variables significantly associated with HR in univariate correlation analysis were entered as independent variables in a linear multiple regression analysis predicting HR. The associations between HR and time to CVE were analyzed using the Cox proportional hazards regression model. All Cox models were stratified by Center. In addition, since patients within the same site may share similar hidden risk factors, we used Cox proportional hazards models with shared frailty, where cluster effects were incorporated into the models as an independent and identically distributed (in our case gamma distributed) random variable (23). The hazard ratios of the univariate and multivariable analyses and corresponding two-sided $95 \%$ confidence intervals were derived from the regression coefficients in the Cox models. Hazard ratios were adjusted for age, BMI, blood pressure, and total serum cholesterol and creatinine concentrations, which were fitted as continuous variables, and for gender, smoking, alcohol intake and diabetes, which were fitted as categorical variables. Adjusted models for the night:day HR ratio additionally included the corresponding night:day systolic blood pressure ratio and average 24-hour HR. All available risk factors were entered into a first model. A parsimonious set of covariates was selected using sequential backward elimination of the least significant variables. Subsequently, a final model was developed in which the HR was entered as the first variable and then was adjusted for all the other variables that were found to be significant (final parsimonious model). To compare non-nested models, we used the Akaike Information Criteria (AIC) and the Schwarz's Bayesian Information Criterion (BIC) $(24,25)$. For both AIC and BIC criteria, the model providing smallest values is considered to have the best fit $(24,25)$. In addition, a measure that can be used to compare survival models is the delta AIC $(\Delta \mathrm{i})$. This is a measure of each model AIC relative to the model with the smallest AIC. As a rule for interpretation, a $\Delta \mathrm{i}<2$ suggests substantial evidence for the model, values between 3 and 7 indicate that the model has considerably less support, whereas a $\Delta \mathrm{i}>10$ indicates that the model is very unlikely (26). The risk of cardiovascular events as a function of nighttime HR was tested in relation to night-time systolic blood pressure in the subjects stratified 
by gender. In this analysis, night-time HR was represented by four risk functions corresponding to levels of 55, 62, 68, and 77 beats/min (midpoints within each HR quartile).

Analyses were performed using a significance level of $a=.05$ (2-sided). To ensure the robustness of the present findings, 4 sensitivity analyses were performed, excluding subjects who experienced an event within 1,2,3, and 5 years of enrollment. As medication with beta-blockers may influence HR during the follow-up, we also made an analysis after excluding the 996 patients known to be taking beta-blockers after baseline assessment. In all outcome analyses only the first event was considered.

\section{RESULTS}

The study population consisted of 3435 women (45.2\%) and 4165 men (54.8\%). Of these, 6583 were Caucasians (86.6\%) and 1017 were Asians (13.4\%). None of the subjects considered for this analysis had atrial fibrillation or other relevant arrhythmias at baseline assessment. At enrollment, 1556 participants (20.5\%) were current smokers, and 608 (8.0\%) were diabetics. Mean age \pm SD was $52.4 \pm 15.8$ years, office systolic blood pressure was $155.7 \pm 16.5 \mathrm{~mm} \mathrm{Hg}$, diastolic BP was $95.0 \pm 9.5 \mathrm{~mm} \mathrm{Hg}$, and body mass index (BMI) was $26.2 \pm 3.9 \mathrm{~kg} / \mathrm{m}^{2}$. Office HR $(76.4 \pm 11.1 \mathrm{bpm})$ was higher than average 24-hour HR $(73.7 \pm 8.8$ beats/min). Ambulatory HR averaged $77.4 \pm 9.4$ and $65.5 \pm 9.0$ beats/min during daytime and night-time, respectively. Age-adjusted night-time HR was higher in women than men $(67.2 \pm 8.9$ vs $64.1 \pm 8.9$ beats $/$ min, $p<0.001)$, in smokers than non smokers $(67.0 \pm 9.7$ vs $65.1 \pm 8.8$ beats $/ \mathrm{min}, \mathrm{p}<0.001)$, and in diabetic than non diabetic patients $(66.9 \pm 8.9 \mathrm{vs}$ $65.3 \pm 10.1$ beats/min, $p<0.001$ ). In simple regression analyses, the night-time HR showed significant associations with age (negative), BMI, night-time systolic and diastolic blood pressures, glucose, total cholesterol, and creatinine (all $\mathrm{p}<0.001$ ). In the subsequent multivariable analysis, the nighttime HR was negatively associated with age $(\mathrm{p}<0.001)$ and was positively associated with female gender, BMI, night-time systolic or diastolic blood pressure, glucose, cholesterol, diabetes, and smoking (all $\mathrm{p}<0.001$ ).

\section{Cardiovascular events}

During a median follow-up of 5.0 years (interquartile range, 3.5-8.4 years), there were 625 fatal plus nonfatal CVE and 14 cases with end-stage renal disease. Considering causespecific first CVE, the incidence of fatal and nonfatal stroke amounted to 37 and 222, respectively. Cardiac events included 18 fatal and 145 nonfatal cases of acute myocardial infarction, 25 sudden deaths, 10 deaths from other cardiovascular causes, 59 cases of heart failure requiring hospitalization, 64 cases of surgical or percutaneous coronary revascularization, and 45 cases of peripheral vascular disease.

\section{Risk associated with increased heart rate}

In Cox models adjusted for age and sex and stratified by Center, a 10 beats/min increase in each of the baseline ambulatory HR measures was significantly associated with increased CVE risk (table 1). Among the ambulatory HRs, the risk of CVE was higher for night-time HR than for 24-hour HR or daytime HR. Night-time HR consistently predicted cardiac events ( $\mathrm{p}=0.001)$, fatal and nonfatal myocardial infarction $(\mathrm{p}=0.032)$, and fatal and nonfatal stroke ( $\mathrm{p}=0.002$ ). Office HR was a weaker predictor of outcome than the ambulatory HRs. Inclusion of systolic and diastolic blood pressures as covariates in the model attenuated the relationship of the ambulatory HRs with CVE and office HR was no longer a significant predictor of outcome in this model (table 1). All ambulatory systolic blood pressures and office systolic blood pressure were strong predictors of outcome $(\mathrm{p}<0.001)$ whereas all diastolic blood pressures were not accepted in the models. BMI $(\mathrm{p}=0.70)$ and alcohol consumption $(\mathrm{p}=0.45)$ were other variables not accepted in the models and were not 
included in the final parsimonious models. The multivariate-adjusted hazard ratios for CVE associated with a 10 beats/min increase in the ambulatory HRs are shown in table 1 . In these models, night-time HR and 24-hour HR remained significant predictors of outcome while the association was no longer significant for daytime HR. The model including night-time HR had the smallest AIC, and neither the model including daytime HR nor that including 24-hour HR had a $\Delta \mathrm{i}<2$ (table 2). Compared to the basic model with covariates, BIC was higher for either model including daytime HR or 24-hour HR, while the model including night-time HR had the smallest BIC (table 2) lending further support to the latter as being the most informative model. After further adjustment for beta-blocker treatment the p-value for night-time HR was 0.006 . Including a random frailty term in the survival models had little effect on risk estimates and statistical significance of the fixed effects (for $10 \mathrm{bpm}$ increase night-time HR the adjusted hazard ratio was 1.14 and 95\%CI, 1.04-1.25; $\mathrm{p}=0.004$ ) with a very low estimated variance of the random effect (theta $=0.09$ ) corresponding to a Kendall's Tau of 0.043 (a global measure of dependence; values interpreted as $0=$ no correlation; $1=$ =maximum correlation), which reveals a likely negligible dependence. Nighttime HR $(\mathrm{p}=0.040)$ but not 24 -hour HR $(\mathrm{p}=0.16)$ or daytime HR $(\mathrm{p}=0.27)$ remained a significant predictor of outcome also when office HR was forced in the Cox model. The night:day ratio of HR was a significant predictor of CVE after adjustment for age, sex, and average 24-hour HR (table 1). However, when average 24-hour systolic blood pressure and the night:day ratio of systolic blood pressure were included in the Cox model this association was no longer significant. Night-time HR remained a significant predictor of outcome in a sensitivity analysis from which we excluded patients who were treated with beta-blockers during the follow-up (table 3). In contrast with the overall result, no relationship between night-time HR and cardiovascular outcomes was found within the 996 patients on beta-blockers (hazard ratio, 1.00 and $95 \% \mathrm{CI}, 0.97-1.03 ; \mathrm{p}=0.96$ ). The strength of the association between night-time HR and CVE remained consistent or even increased when we excluded subjects who experienced an event within 1 to 5 years of enrollment (figure 2). Figure 3 shows the independent multivariate-adjusted associations of CVE with night-time systolic blood pressure and night-time HR by gender. The risk of CVE progressively increased with increasing systolic blood pressure and HR in both genders without interaction between sex, systolic blood pressure and HR ( $\mathrm{p}=0.82)$.

\section{DISCUSSION}

The present analysis relating to a cohort of untreated hypertensive patients from the ABPInternational database has demonstrated an independent association of ambulatory HR with fatal combined with nonfatal CVE in these patients. This relationship was linear and similar in the two genders. Among the ambulatory HRs, the model including night-time HR was more informative in predicting the outcome than models with daytime or 24-hour HR. For every 10 -beats/min increment in night-time HR, the adjusted risk of CVE increased by $13 \%$.

\section{Previous studies}

HR measured at rest in the clinic (1-7) or at home (27) has been shown to be an important predictor of adverse cardiovascular outcomes in previous studies. Much less is known about the predictive power of HR recorded over the 24 hours. Results from two general population studies have shown that neither daytime nor night-time HR predicted cardiovascular disease mortality $(12,13)$. However, in the Ohasama study ambulatory HR predicted noncardiovascular disease mortality (12). In a recent analysis of 6 general population studies, 24-hour HR predicted total and noncardiovascular mortality but neither cardiovascular mortality nor any of the fatal combined with nonfatal CVE (13). Previous studies have shown that the predictive power of elevated office HR for cardiovascular disease is greater in hypertensive than in normotensive subjects suggesting that $\mathrm{HR}$ and 
blood pressure may act synergistically in the development of CVE $(28,29)$. To our knowledge, the prognostic significance of ambulatory HR in hypertensive patients has been evaluated in four previous studies. In 393 elderly patients with isolated systolic hypertension and 457 Japanese hypertensives, no independent association of average ambulatory HRs with cardiovascular outcomes was found $(9,14)$. Also in a study by Verdecchia et al in 1942 untreated hypertensive patients ambulatory HR was unrelated to combined fatal and nonfatal CVE (8). Finally, in 3957 referred patients, $50 \%$ of whom were on antihypertensive treatment, Ben-Dov et al found that nocturnal HR but not daytime HR was associated with total mortality (11). Unfortunately, in the Ben-Dov et al study no information was available about CVE.

\section{The present study}

The present results were obtained in the largest ever study of hypertensive patients assessed with ambulatory HR monitoring. All patients were untreated at the time of baseline assessment thereby avoiding the confounding effect of HR lowering drugs. However, pharmacological manipulation of HR during the follow-up might have altered the relationship between baseline HR and outcome (30). For this reason we repeated the Cox analysis after excluding the 996 subjects taking beta-blockers during the follow-up period obtaining consistent results, with an adjusted increase in CVE risk of 15\% per 10-beats/min increment in night-time HR. In contrast with this finding, within the subjects taking betablockers during the follow-up period no relation at all was found between night-time HR and cardiovascular outcomes $(\mathrm{p}=0.96)$ indicating that after pharmacological HR reduction the association of pre-treatment HR with subsequent CVE can be lost.

In the present study, among the ambulatory HRs, night-time HR showed a better predictive value than daytime HR. This finding is in keeping with previous data by others which showed that high HR during the night-time but not during waking hours was associated with increased noncardiovascular mortality $(12,13)$ and raises the question about the pathophysiological significance of elevated HR during sleep.

\section{Mechanisms}

A possible mechanism by which a fast HR might relate to CVE is through increased sympathetic tone having adverse hemodynamic effects which might accelerate atherosclerosis (31-34). HR during sleep is more stable and reproducible than HR during waking hours which is subject to random fluctuations related to physical activities and occasional emotional triggers. Daytime HR may be less reproducible also across different centers because of the differing procedures used during the recordings (standardized versus non-standardized activities). In a study by Perski et al on the relationship between ambulatory HR and coronary atherosclerosis, minimum HR recorded during the 24-hour period was the strongest predictor of severity of diffuse coronary atherosclerosis whereas maximum HR and the total number of heart beats during the 24 hours were not related to outcome (35). The above considerations can also explain the closer association of cardiovascular outcomes with night-time HR compared to office HR. The studies included in the present analysis were not specifically designed to assess the prognostic value of office $\mathrm{HR}$, and thus this variable was not recorded in a standardized fashion across the centers. This methodological pitfall is well recognized $(13,36)$ but it may not be the only explanation for the different association of office HR and night-time HR with cardiovascular outcomes. As shown by our previous results, office HR is correlated with the hemodynamic reaction elicited by the doctor's visit reflecting sympathetic nervous system reactivity and is thus poorly representative of the basal HR (35). HR during sleep is more representative of the overall hemodynamic load on the arteries and the heart. Thus a high sleeping HR would better reflect cumulative arterial injury from mechanical stress on the arterial wall. In 
addition, persistent sympathetic over-activity may be better represented by an elevated sleeping HR than by office HR (11). Indeed, in the present study we found strong correlations of night-time HR with blood pressure, BMI, and metabolic abnormalities which may be explained by a high sympathetic tone underlying elevated HR (31-34). In addition, a high sleep HR may reflect episodes of sleep apnea that are associated with an increase in sympathetic drive (37). The better predictive power of night-time HR compared with daytime HR, found in the present study, parallels previous data on ambulatory blood pressure, which showed a stronger association of blood pressure during sleep than during waking hours with CVE (15).

\section{Day-night HR dip}

Whether night-time HR itself or the extent of the day-night dip is important for predicting CVE remains controversial. In some studies, the day-night HR dip ratio has been found to be significantly related to cardiovascular outcomes and to non-cardiovascular mortality (8,11-14). However, when average ambulatory HR was included into the same model, the day-night HR dip ratio was no longer predictive of cardiovascular outcomes in the studies by Hansen et al. (13) and by Verdecchia et al (8), or of non-cardiovascular mortality in the Ohasama study (12). In the present study, the association of the HR dip with CVE ceased to be significant when average night-time systolic BP and/or the night:day ratio of systolic BP was included in the Cox model, confirming that sleep HR itself rather than the dip ratio is important for predicting cardiovascular morbidity, as sleep HR remained an independent predictor when these variables were included in the model.

There is still controversy over whether the association of resting HR with cardiovascular outcomes is similar for coronary events and stroke. Previous studies suggested that the association of resting office HR with cardiovascular disease was mainly due to a strong association with coronary heart disease rather than with cerebrovascular events $(1,38)$ although some studies found a similar impact of high office HR on coronary and cerebrovascular mortalities $(39,40)$. The present results obtained with ambulatory monitoring indicate that night-time HR is an independent predictor of both coronary events and stroke.

\section{Limitations}

Although we showed that high ambulatory HR was a predictor of CVE, the association between high HR and CVE might be explained by reverse causality. In some patients a high HR at night might be due to a chronic disorder that was not clinically manifest at the time of baseline assessment. In that case, an elevated HR should be considered as an indicator of poor physical health. In the present study, we could not adjust for important determinants of HR, such as hemoglobin concentration, or usual physical activity. However, the association between high HR and risk of CVE remained robust and even increased when patients who experienced an event during the first 5 years of follow-up were eliminated, thereby minimizing the possibility of high HR being the consequence rather than the cause of CVE.

\section{Conclusion}

We found that HR recorded during sleep with ambulatory monitoring adds prognostic information in hypertensive patients beyond that of established ambulatory blood pressure parameters. Thus, ambulatory HR might be considered by the practicing physician as an additional tool for cardiovascular risk stratification. Whether lowering high ambulatory HR in hypertensive patients can improve cardiovascular outcomes remains to be seen. 


\title{
Acknowledgments
}

\author{
Sources of funding: none
}

The authors of this manuscript have certified that they comply with the Principles of Ethical Publishing in the International Journal of Cardiology (41).

\section{References}

1. Kannel WB, Kannel C, Paffenbarger RS Jr, Cupples LA. Heart rate and cardiovascular mortality: The Framingham Study. Am Heart J. 1987; 113:1489-1494. [PubMed: 3591616]

2. Palatini P, Casiglia E, Julius S, Pessina AC. High heart rate: a risk factor for cardiovascular death in elderly men. Arch Intern Med. 1999; 159:585-592. [PubMed: 10090115]

3. Jouven X, Empana JP, Schwartz PJ, Desnos M, Courbon D, Ducimetiere P. Heart-rate profile during exercise as a predictor of sudden death. N Engl J Med. 2005; 352:1951-1958. [PubMed: 15888695]

4. Kolloch R, Legler UF, Champion A, et al. Impact of resting heart rate on outcomes in hypertensive patients with coronary artery disease: findings from the INternational VErapamil-SR/trandolapril STudy (INVEST). Eur Heart J. 2008; 29:1327-1334. [PubMed: 18375982]

5. Paul L, Hastie CE, Li WS, et al. Resting Heart Rate Pattern During Follow-Up and Mortality in Hypertensive Patients. Hypertension. 2010; 55:567-574. [PubMed: 20038750]

6. Copie X, Hnatkova K, Staunton A, Fei L, Camm AJ, Malik M. Predictive power of increased heart rate versus depressed left ventricular ejection fraction and heart rate variability for risk stratification after myocardial infarction. Results of a two-year follow-up study. J Am Coll Cardiol. 1996; 27:270-276. [PubMed: 8557893]

7. Diaz A, Bourassa MG, Guertin MC, Tardif JC. Long-term prognostic value of resting heart rate in patients with suspected or proven coronary artery disease. Eur Heart J. 2005; 26:967-974. [PubMed: 15774493]

8. Verdecchia P, Schillaci G, Borgioni C, et al. Adverse prognostic value of a blunted circadian rhythm of heart rate in essential hypertension. J Hypertens. 1998; 16:1335-1343. [PubMed: 9746121]

9. Palatini P, Thijs L, Staessen JA, et al. Predictive value of clinic and ambulatory heart rate for mortality in elderly subjects with systolic hypertension. Arch Intern Med. 2002; 162:2313-2321. [PubMed: 12418945]

10. Sega R, Facchetti R, Bombelli M, et al. Prognostic value of ambulatory and home blood pressures compared with office blood pressure in the general population: follow-up results from the Pressioni Arteriose Monitorate e Loro Associazioni (PAMELA) study. Circulation. 2005; 111:1777-1783. [PubMed: 15809377]

11. Ben-Dov IZ, Kark JD, Ben-Ishay D, Mekler J, Ben-Arie L, Bursztyn M. Blunted heart rate dip during sleep and all-cause mortality. Arch Intern Med. 2007; 167:2116-2121. [PubMed: 17954807]

12. Hozawa A, Inoue R, Ohkubo T, et al. Predictive value of ambulatory heart rate in the Japanese general population: The Ohasama study. J Hypertens. 2008; 26:1571-1576. [PubMed: 18622234]

13. Hansen TW, Thijs L, Boggia J, et al. Prognostic value of ambulatory heart rate revisited in 6928 subjects from 6 populations. Hypertension. 2008; 52:229-235. [PubMed: 18574073]

14. Eguchi K, Hoshide S, Hishikawa J, et al. Nocturnal nondipping of heart rate predicts cardiovascular events in hypertensive patients. J Hypertens. 2009; 27:2265, 2270. [PubMed: 19834343]

15. Staessen JA, Thijs L, Fagard R, et al. Predicting cardiovascular risk using conventional vs ambulatory blood pressure in older patients with systolic hypertension. Systolic Hypertension in Europe Trial Investigators. JAMA. 1999; 282:539-546. [PubMed: 10450715]

16. Verdecchia P, Porcellati C, Schillaci G, et al. Ambulatory blood pressure: an independent predictor of prognosis in essential hypertension. Hypertension. 1994; 24:793-801. [PubMed: 7995639]

17. Kario K, Shimada K, Schwartz JE, Matsuo T, Hoshide S, Pickering TG. Silent and clinically overt stroke in older Japanese subjects with white-coat and sustained hypertension. J Am Coll Cardiol. 2001; 38:238-245. [PubMed: 11451281] 
18. Pierdomenico SD, Di Nicola M, Esposito AL, et al. Prognostic value of different indices of blood pressure variability in hypertensive patients. Am J Hypertens. 2009; 22:842-847. [PubMed: 19498342]

19. Wing LM, Brown MA, Beilin LJ, Ryan P, Reid CM. ANBP2 Management Committee and Investigators. Second Autralian National Blood Pressure Study. 'Reverse white-coat hypertension' in older hypertensives. J Hypertens. 2002; 20:639-644. [PubMed: 11910298]

20. Eguchi K, Kario K, Shimada K. Greater impact of coexistence of hypertension and diabetes on silent cerebral infarcts. Stroke. 2003; 34:2471-2474. [PubMed: 14500939]

21. Palatini P, Winnicki M, Santonastaso M, et al. Prevalence and clinical significance of isolated ambulatory hypertension in young subjects screened for stage 1 hypertension. Hypertension. 2004; 44:170-174. [PubMed: 15210653]

22. Winnicki M, Somers VK, Dorigatti F, et al. Lifestyle, family history and progression of hypertension. J Hypertens. 2006; 24:1479-1487. [PubMed: 16877948]

23. Oakes D. Bivariate Survival Models Induced by Frailties. J Am Stat Assoc. 1989; 84:487-493.

24. Akaike H. A new look at the statistical model identification. IEEE Trans Automat Contr. 1974; 19:716-723.

25. Schwarz G. Estimating the dimension of a model. Ann Stat. 1978; 6:461-464.

26. Burnham, KP.; Anderson, DR. Model Selection and Multimodel Inference: a practical informationtheoretic approach. 2. Springer-Verlag; New York, NY, USA: 2002.

27. Hozawa A, Ohkubo T, Kikuya M, et al. Prognostic value of home heart rate for cardiovascular mortality in the general population: the Ohasama study. Am J Hypertens. 2004; 17:1005-1010. [PubMed: 15533725]

28. Gillman MW, Kannel WB, Belanger A, D'Agostino RB. Influence of heart rate on mortality among persons with hypertension: The Framingham study. Am Heart J. 1993; 125:1148-1154. [PubMed: 8465742]

29. Benetos A, Rudnichi A, Thomas F, Safar M, Guize L. Influence of heart rate on mortality in a French population: role of age, gender and blood pressure. Hypertension. 1999; 33:44-52. [PubMed: 9931080]

30. Bangalore S, Messerli FH, Kostis JB, Pepine CJ. Cardiovascular protection using beta-blockers: a critical review of the evidence. J Am Coll Cardiol. 2007; 50:563-572. [PubMed: 17692739]

31. Palatini P, Julius S. Heart rate and the cardiovascular risk. J Hypertens. 1997; 15:3-17. [PubMed: 9050965]

32. Reil JC, Bohm M. The role of heart rate in the development of cardiovascular disease. Clin Res Cardiol. 2007; 96:585-592. [PubMed: 17593316]

33. Giannoglou GD, Chatzizisis YS, Zamboulis C, et al. Elevated heart rate and atherosclerosis: an overview of the pathogenetic mechanisms javascript:AL_get(this, 'jour', 'Int J Cardiol.'); Int J Cardiol. 2008; 126:302-312. [PubMed: 18068835]

34. Fox KM, Ferrari R. Heart rate: a forgotten link in coronary artery disease? Nat Rev Cardiol. 2011; 8:369-379. [PubMed: 21519356]

35. Perski A, Olsson G, Landou C, et al. Minimum heart rate and coronary atherosclerosis: Independent relations to global severity and rate of progression of angiographic lesions in men with myocardial infarction at a young age. Am Heart J. 1992; 123:609-616. [PubMed: 1539512]

36. Palatini P, Winnicki M, Santonastaso M, et al. Reproducibility of heart rate measured in the clinic and with 24-hour intermittent recorders. Am J Hypertens. 2000; 13:92-98. [PubMed: 10678277]

37. Somers VK, Dyken ME, Clary MP, Abboud FM. Sympathetic neural mechanisms in obstructive sleep apnea. J Clin Invest. 1995; 96:1897-1904. [PubMed: 7560081]

38. Okamura T, Hayakawa T, Kadowaki T, et al. Resting heart rate and cause-specific death in a 16. 5year cohort study of the Japanese general population. Am Heart J. 2004; 147:1024-1032. [PubMed: 15199351]

39. Thomas F, Bean K, Provost JC, Guize L, Benetos A. Combined effects of pulse pressure and heart rate on cardiovascular mortality. J Hypertens. 2001; 19:863-869. [PubMed: 11393668] 
40. Sandvik L, Erikssen J, Ellestad M, et al. Heart rate increase and maximal heart rate during exercise as predictors of cardiovascular mortality: a 16-year follow-up study of 1960 healthy men. Coron Artery Dis. 1995; 6:667-679. [PubMed: 8574463]

41. Coats AJS, Shewan LG. Statement on Authorship and Publishing Ethics in the International Journal of Cardiology. Int J Cardiol. 2011; 153:239-40. [PubMed: 22108502] 


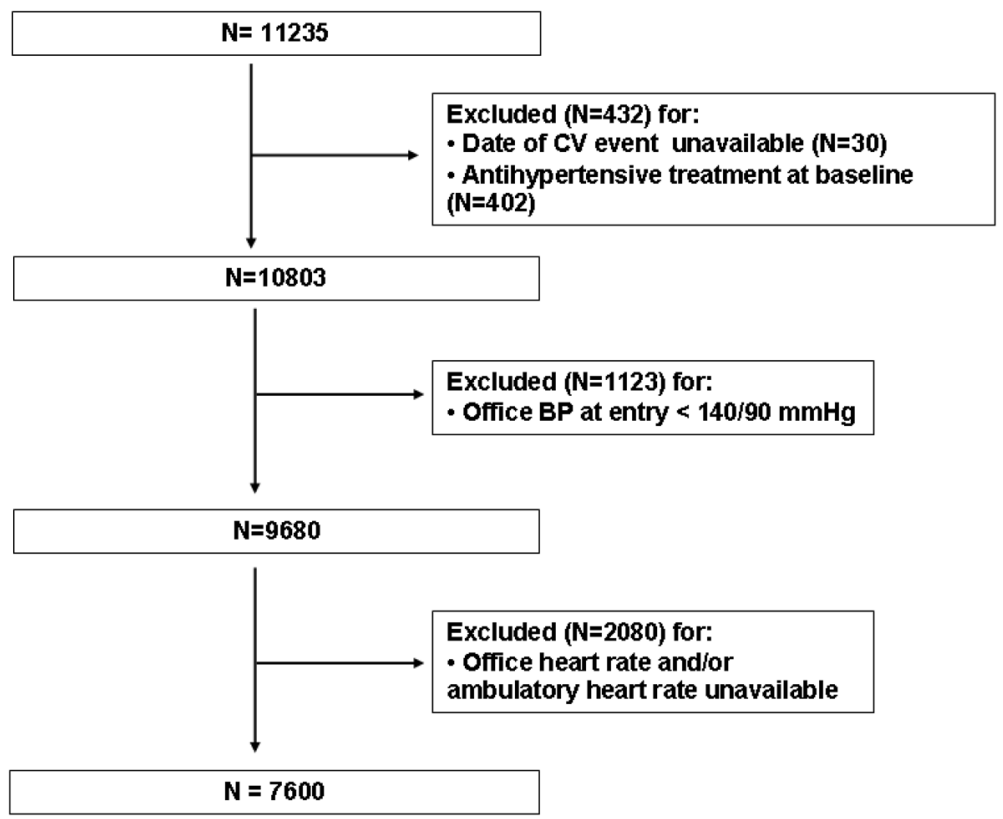

Figure 1.

Flow-chart of the patients from the ABP-International study selected for the present analysis. 


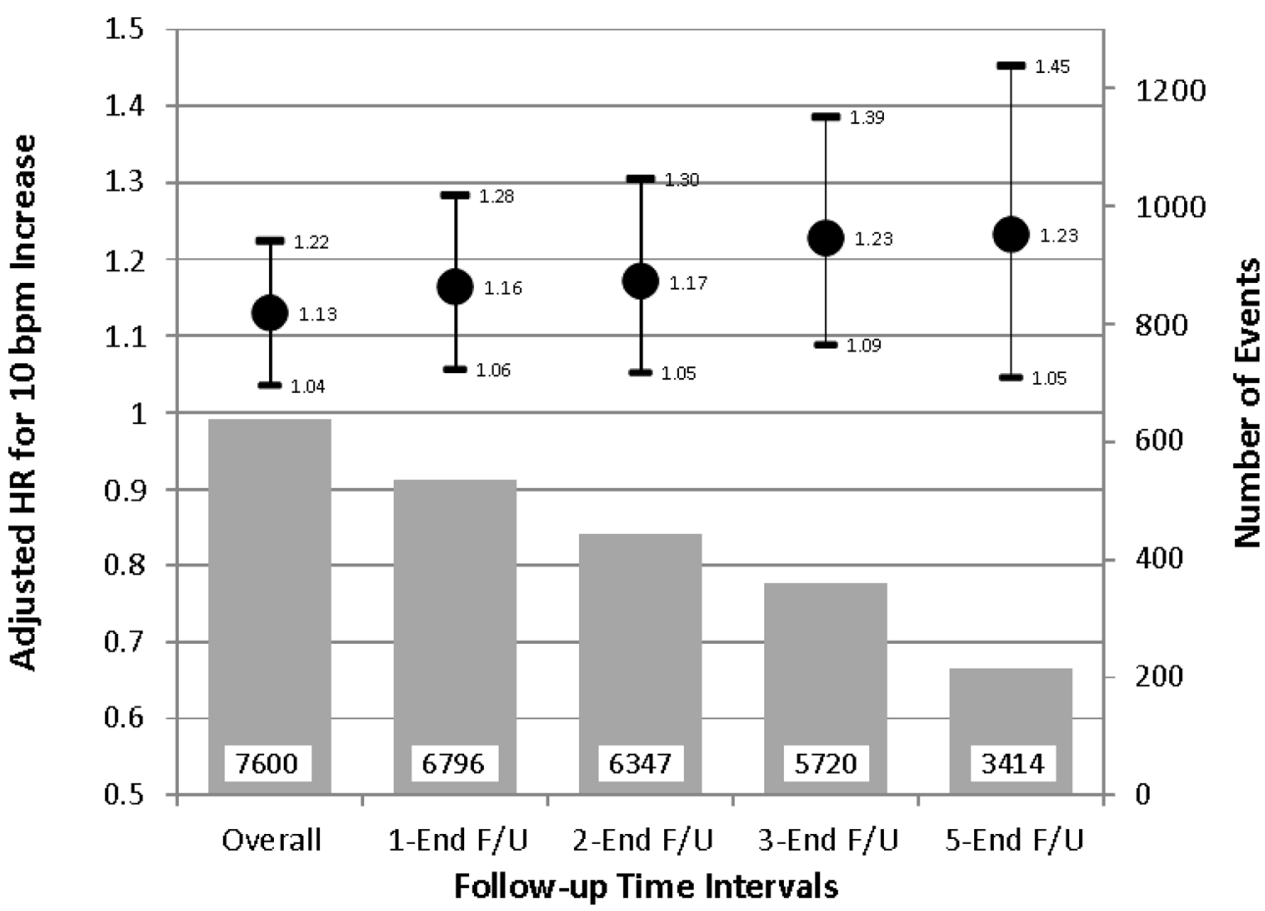

Figure 2.

Risks of cardiovascular events and $95 \%$ confidence intervals from sensitivity analyses in which subjects who experienced a cardiovascular event within 1 year, 2 years, 3 years, or 5 years from baseline assessment were progressively excluded. Using the Cox model, the hazard ratio is estimated by excluding all observations corresponding to survival times before the lower time interval point. Circles indicate adjusted hazard ratios for a $10 \mathrm{bpm}$ increment in night-time heart rate. Bars indicate number of events. Numbers in the bars indicate number of participants at risk. 1-End F/U indicates from year 1 to end of follow-up. Risk estimates were adjusted for cohort, age, gender, smoking, diabetes, night-time systolic blood pressure, and serum total cholesterol and serum creatinine concentrations. 

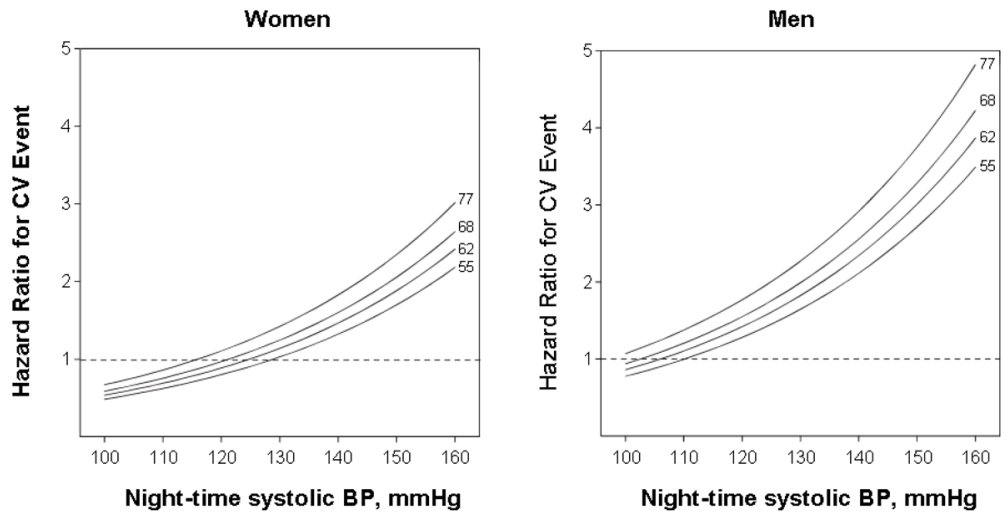

Figure 3.

Hazard ratio of fatal and nonfatal combined cardiovascular events associated with nighttime systolic blood pressure and night-time heart rate in 7600 hypertensive individuals stratified by gender. The night-time systolic blood pressure is plotted along the $\mathrm{x}$-axis and the scale covers the $5^{\text {th }}$ to $95^{\text {th }}$ percentile interval. The night-time heart rate is represented by four functions corresponding to levels of 55, 62, 68, and 77 beats/min (midpoints within each heart rate quartile). Risk estimates were adjusted to 52 years of age, non-smoking, lack of diabetes, $206 \mathrm{mg} / \mathrm{dl}$ total cholesterol, and $0.9 \mathrm{mg} / \mathrm{dl}$ serum creatinine. P-value for systolic blood pressure $<0.001$, P-value for heart rate $=0.007$. The interaction term between the night-time systolic blood pressure and heart rate and gender was not significant $(\mathrm{p}=0.82)$. 


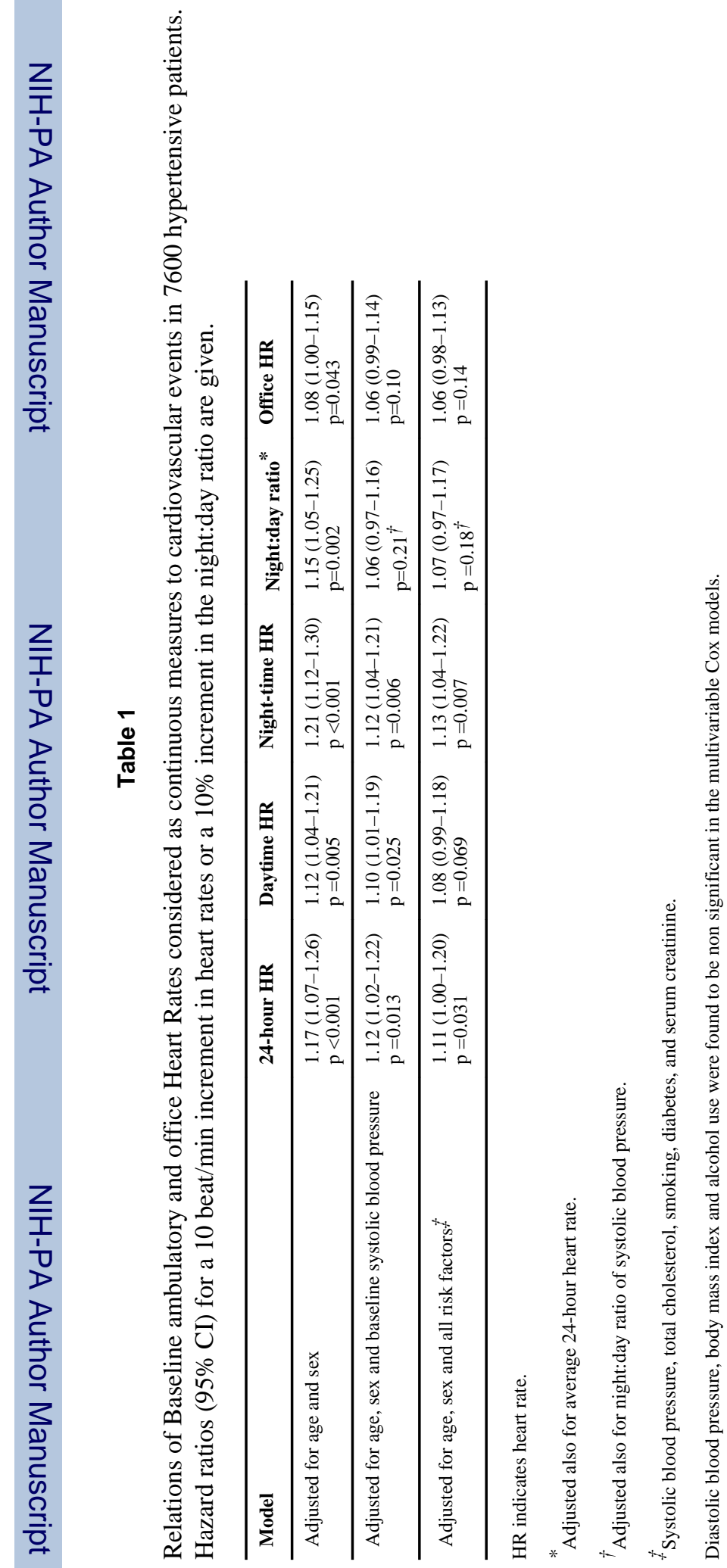




\section{Table 2}

AIC, Delta AIC, and BIC for the different Cox models.

\begin{tabular}{cccc}
\hline Models & AIC & & BIC \\
\hline Empty Model & 8146.365 & Delta AIC & 8146.365 \\
Base Model $^{*}(\mathrm{M} 0)$ & 7495.757 & 5.953 & 7526.629 \\
Model 1 (M1): & 7494.129 & 4.325 & 7529.411 \\
M0 + Daytime HR & & & \\
Model 2 (M2): & 7493.146 & 3.342 & 7528.427 \\
M0 + 24-hour HR & & & \\
$\begin{array}{c}\text { Model 3 (M3): } \\
\text { M0 + Night-time HR }\end{array}$ & 7489.804 & minAIC & 7525.085 \\
\hline * & & \\
Including: Age, Gender, Diabetes, Smoking, Cholesterol, Creatinine, Systolic blood pressure
\end{tabular}

AIC indicates Akaike Information Criterion; Delta AIC is a measure of each model relative to the model with the smallest AIC (minAIC); BIC indicates Bayesian Information Criterion; HR, heart rate. 


\section{Table 3}

Multivariable Cox analysis of risk function for fatal and nonfatal cardiovascular events in 6604 hypertensive patients not taking beta-blockers during the follow-up.

\begin{tabular}{lcc}
\hline Variable & Hazard ratio (95\% CI) & p-value \\
\hline Night-time heart rate (per 10-beat/min increase) & $1.15(1.05-1.25)$ & .0069 \\
Male sex & $1.68(1.38-2.03)$ & $<.001$ \\
Age (per 10-year increase) & $1.71(1.61-1.81)$ & $<.001$ \\
Smoking (yes/no*) & $1.64(1.34-2.00)$ & $<.001$ \\
Diabetes mellitus (yes/no $\left.{ }^{*}\right)$ & $1.30(1.02-1.66)$ & .03 \\
Night-time systolic BP (per 10-mm Hg increase) & $1.27(1.22-1.32)$ & $<.001$ \\
Total cholesterol (per 10-mg/dL increase) & $1.04(1.02-1.06)$ & $<.001$ \\
Serum creatinine (per 0.1-mg/dL increase) & $1.08(1.05-1.11)$ & $<.001$ \\
\hline * & &
\end{tabular}

BP indicates blood pressure; alcohol use, body mass index, and diastolic blood pressure were not accepted by the model ( $p>0.46)$. 